\title{
MAGNETO-OPTICAL EFFECT OF Co-SUBSTITUTED YTTRIUM NEODYMIUM IRON GARNET FILM IN VISIBLE AND NEAR INFRARED REGIONS
}

\author{
Y. TORIUMI, A. ITOH, E. MIZOBUCHI, K. KATAYAMA, F. INOUE, \\ and K. KAWANISHI \\ Dept. of Electronic Engn., College of Sci. \& Tech., Nihon University, Narashino-dai, Funabashi, Chiba 274, Japan
}

\section{ABSTRACT}

Dielectric tensor of Co, Ge-doped YNdIG films grown on GGG by LPE method were derived in the range of $0.5 \mu \mathrm{m}$ to $1.8 \mathrm{\mu m}$. On the assumptions of Lorentzian line shape and the existence of three optical transitions at $1.5 \mu \mathrm{m}$ band, splitting width and half width of the transitions are derived through the parameter fitting procedure. They are constant against co content of the films. Therefore, the optical figure of merit at $1.5 \mu \mathrm{m}$ is constant against divalent Co content. Also shown is the spectra of dielectric tensor of trivalent co-doped YbSmIG.

\section{INTRODUCTION}

Garnet ferrite is known as one of the most useful material for magneto-optical devices and memories. Enhancement of magneto-optical effect as well as decrease of optical absorption are the important problems for such applications.

Co-substitution in garnet films with tetravalent charge compensation ions enhances strongly the Faraday rotation $\left(\theta_{\mathrm{F}}\right)$ at $0.6 \mu \mathrm{m}(2.0 \mathrm{eV})$ and $1.5 \mu \mathrm{m}(0.8 \mathrm{eV})$ bands while trivalent co ion enhances only at $1.3 \mu \mathrm{m}(0.95 \mathrm{eV})$. Egashira et al. ${ }^{[1]}$ reported the experimental results of the effect of divalent and trivalent Co ions on Faraday rotation coefficient of bulk crystals of GdIG. Pappalardo et al. ${ }^{[2]}$ studied the optical absorption spectra of Co-doped yttrium gallium garnet. They derived oscillator strength and cubic-field parameter.

Co concentration dependence of magneto-optical figure of merit of co doped garnet films also offers an interesting problem from the viewpoint of application to optical devices. Crystal field transitions of tetrahedral $C_{0}$ ions contribute to these effects. Estimation of dielectric tensor is useful in the discussion of the magneto-optical effect.

In this paper, we report the off-diagonal elements of dielectric tensor of divalent co substituted iron garnet films at photon energies below absorption edge $(0.5-1.8 \mu \mathrm{m},(0.7-2.3 \mathrm{eV}))$. The film was grown on GGG (111) oriented substrate by the LPE method. On an assumption of Lorentzian line shape, halfwidth $\left(\Gamma_{0}\right)$ at half-height and splitting width of the transition are derived, and their dependence on Co concentration is discussed. The results of measurements of the dielectric tensor for trivalent Co doped YbSmIG are also given and discussed.

\section{EXPERIMENTAL AND ANALYSIS METHOD}

\section{L.Growth condition}

$\mathrm{Y}_{2 .}{ }_{9} \mathrm{Ndo}_{1} \mathrm{Cox}_{\mathrm{x}} \mathrm{Ge}_{y} \mathrm{Fe}_{5-x-y} \mathrm{O}_{12}$ films were grown on the GGG (111) oriented substrates by the LPE method from a $\mathrm{PbO}-\mathrm{B}_{2} \mathrm{O}_{3}$ fluxed melt. Nd is used for control of lattice constant. Tetravalent Ge ions are incorporated as charge compensation ions of $\mathrm{Co}_{0} 2+$ in the films. When the $\mathrm{CO}$ and Ge concentrations (X and $Y$ ) are below 1 mol/f.u., and the content of Nd is 0.1 , lattice mismatch between the films and the substrates is in the range from 0 to $1 \%$. For controlling $C_{0}$ and Ge concentrations in the films, the additional parameters of the melt $R_{2}{ }^{\prime}$ and $R_{2}{ }^{\prime \prime}$ are defined as $R_{q}{ }^{\prime}=2\left(\mathrm{Fe}_{2} \mathrm{O}_{3}\right) /(\mathrm{COO})$, $\mathrm{R}_{2}^{\prime \prime}=2\left(\mathrm{Fe}_{2} \mathrm{O}_{3}\right) /\left(\mathrm{GeO}_{2}\right)$ and changed systematically.

For doping of trivalent $\mathrm{Co}$, a $\mathrm{PbO}-\mathrm{V}_{2} \mathrm{O}_{5}$ fluxed melt is used and quadrivalent charge compensation ion is not substituted. In the films grown from the melt any trace of $v^{5+}$ ion is not detected.

\section{Determination of dielectric tensor of Co-substituted garnet}

For a geometry with a cubic material and a magnetization parallel to the $Z$ axis, the dielectric tensor has the form given by

$$
\varepsilon=\left[\begin{array}{ccc}
\varepsilon_{0} & +i \varepsilon_{1} & 0 \\
-i \varepsilon_{1} & \varepsilon_{0} & 0 \\
0 & 0 & \varepsilon_{z}
\end{array}\right]
$$

Each element has a real and a imaginary part as $\varepsilon_{i}=\varepsilon i+i \varepsilon i$

The relation between the off-diagonal elements, the optical constants(n:refractive index, $k$ :extinction coefficient) and the magneto-optical parameters ( $\theta_{F}:$ Faraday rotation, $\eta_{F}: F$ araday ellipticity) can be expressed by the following equations.

$$
\begin{aligned}
& \varepsilon_{i}=\frac{\lambda}{\pi}\left(\mathrm{n} \theta_{F}-\mathrm{k} n_{F}\right) \\
& \varepsilon_{i}=\frac{\lambda}{\pi}\left(\mathrm{n} n_{F}+k \theta_{F}\right)
\end{aligned}
$$


The optical constants $n$ and $k$ were combined by Kramers-Kronig relation

$$
-\phi\left(\omega_{1}\right)=\frac{1}{2 \pi} \int_{0}^{\infty}(\ln R(\omega)) \cdot \ln \frac{\left|\omega_{1}-\omega\right|}{\left|\omega_{i}+\omega\right|} d \omega
$$

$n$ and $k$ can be derived through the measurement of complex reflection coefficient lamplitude $R$ and phase shift $\phi$ ) and they are given by

$$
\begin{aligned}
& \mathbf{n}=\frac{(1-R)}{1+R-2 \sqrt{R} \cos \phi} \\
& k=\frac{2 \sqrt{R} \sin (-\phi)}{1+R-2 \sqrt{R} \cos \phi}
\end{aligned}
$$

In the above equation (4), the value of reflection $R(\omega)$ as a function of frequency through $\omega=0$ to $\infty$ is necessary to evaluate $n$ and $k$. Usually, some assumptions are made about the functional form of $R(\omega)$ in the higher and lower energy regions as reported by Roessler [3]. Based on the following reasons, we assumed that reflection of Co-substituted garnet is equal to that of $V I G[6]$

(1) Since the films deposited on GGG substrate are thin, it is not easy to determine the acculate reflection coefficient because of the multi-reflection effect in the transparent region.

(2) In the case of Co doped films, at the absorption peak of $1.3 \mu \mathrm{m}(0.95 \mathrm{eV})$ which is the largest local maximum in the range of 0.8-2.0 um, measured absorption coefficient ( $\alpha$ ) is equal to $1100 \mathrm{~cm}^{-1}$ for $x=0.8$. This value is sufficiently small and can be safely neglected. Thus, it is assumed that the extinction coefficient caused by the absorption at $1.3 \mu \mathrm{m}$ is equal to zero.

(3) In the analysis of the dielectric tensor elements of VIG films, Wittecock et al..$^{[4]}$ assumed that the absorption edge of the YIG is equal to $0.5 \mu \mathrm{m}(2.3 \mathrm{eV})$. In the case of Co substituted iron garnet films the influence of the absorption at $1.6 \mu \mathrm{m}(0.7 \mathrm{eV})$ caused by tetrahedral co ions on the value of extinction coefficient $k$ were discussed. When the value of absorption edge is changed from $0.5 \mu \mathrm{m}(2.3 \mathrm{eV})$ to $1.6 \mu \mathrm{m}(0.7 \mathrm{eV})$ in the deriving procedure of $k$ by the method given by Roessler from the equations (4) to $(6)$, the difference in the value of obtained $k$ is only equal to $0.1 \%$.

Magneto-optical parameters $\theta_{F}$ and $\eta_{F}$ are measured with a high frequency polarization modulation method ${ }^{[6]}$ in the range of $0.4 \mu \mathrm{m}(3.1 \mathrm{eV})$ to $1.6 \mu \mathrm{m}(0.7 \mathrm{eV})$. Automatic repetition measurements of magneto-optical spectra are performed with the aid of a microprocessor-based data-acquisition system.

On the basis of the above assumptions and measurements, we have derived the $\epsilon_{1}^{\prime}$ and $\epsilon_{j}^{\prime \prime}$ spectra by the equations from (2) through (6).

\section{RESULTS AND DISCUSSION}

\section{The off-diagonal $\epsilon_{1}$ spectra}

$\epsilon_{1}^{\prime}$ and $\epsilon I^{\prime \prime}$ spectra at $1.6-0.5 \mu \mathrm{m}(0.7-2.3 \mathrm{eV})$ for $\mathrm{CO}^{2+}$ doped films grown on GGG(111) substrates are shown in figures 1 and 2. It is well known that there are two types for optical transitions.

Type 1; The real part $\epsilon y^{\prime}$ shows a bell-shaped behavior passing through zero at $\omega=\omega_{0}$. The imaginary part $\epsilon_{1}$ " shows a dispersive behavior around $\omega_{0}$. These shape are often reffered to as double transitions.

Type 2; The real part $\epsilon_{i}^{\prime}$ has a dispersive shape around $\omega_{0}$ and $\epsilon_{1}^{\prime \prime}$ a dissipative behavior. The $\epsilon_{i}^{\prime}$ curve is often reffered to as single transitions.

E 1 shows a dispersive behavior as shown in Fig. 2 passing through zero near $1.5 \mu m(0.8 \mathrm{eV})$ and $0.6 \mu \mathrm{m}(2.0 \mathrm{eV})$. In Fig. 1, a bell-shaped behavior of $\epsilon i$ is seen at around $1.5 \mu \mathrm{m}$ and $0.6 \mu \mathrm{m}$. From these spectra, transitions at $1.5 \mu \mathrm{m}(0.8 \mathrm{eV})$ and $0.6 \mu \mathrm{m}(2.0 \mathrm{eV})$ are seemed to be the type 1 transitions.

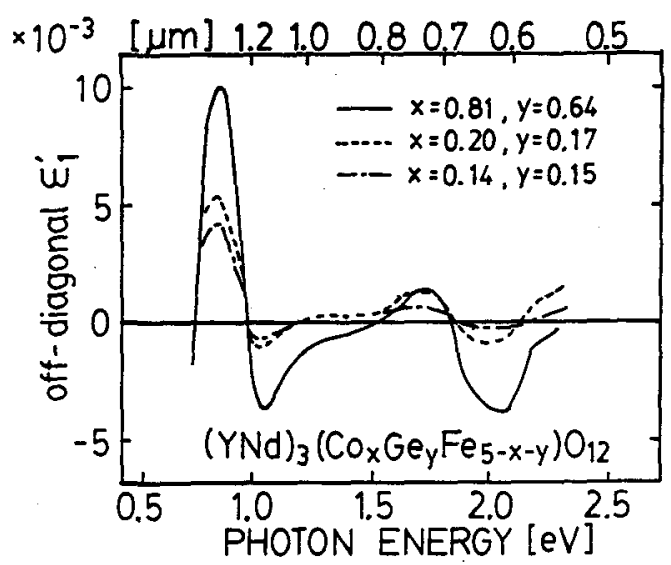

Fig. 1. $\epsilon$ 's spectra of $\mathrm{Y}_{2,9} \mathrm{Ndo}_{1} \mathrm{Co}_{x} \mathrm{Ge}_{y} \mathrm{Fe}_{5-x-y} \mathrm{O}_{12}$ under the variety of $X$ and $Y$.

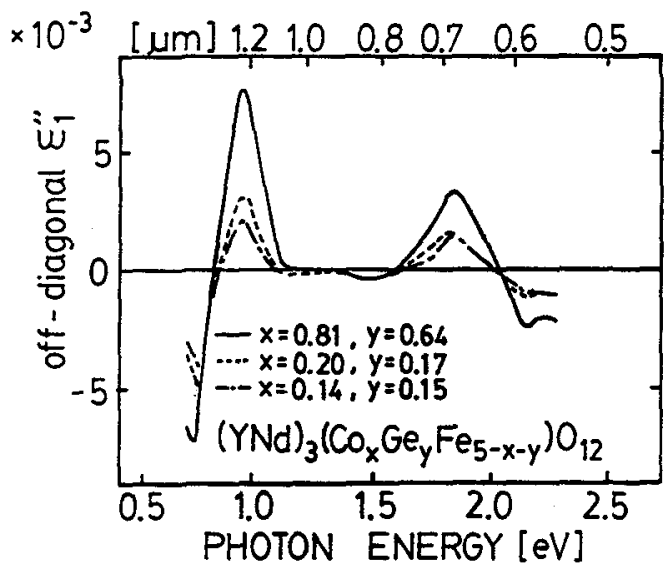

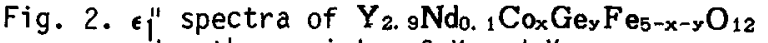
under the variety of $X$ and $Y$. 
The magneto-optical effects at 1.5 and $0.6 \mu \mathrm{m}(0.8$ and $2.0 \mathrm{eV})$ are known as originated from the crystal field effect of divalent co ions in the tetrahedral sites. From the results of the spectra of $\epsilon_{1}^{\prime}$ and $\epsilon_{1}$ ", we determined the value of halfwidth $\left(\Gamma_{0}\right)$ and the splitting width $(\Delta)$ of transitions in the Lorentzian type frequency dependence of $\epsilon_{1}\left(=\epsilon_{1}^{\prime+i} \epsilon_{1}\right)$ of Type 1 transition

$$
\varepsilon_{1}=\frac{\omega_{\mathrm{P}}^{2} \mathrm{f} \Delta L}{2 \omega_{0}} \frac{\left(\omega_{0}-\omega\right)^{2}-\Gamma_{0}^{2}+2 i \Gamma_{0}\left(\omega_{0}-\omega\right)}{\left[\left(\omega_{0}-\omega\right)^{2}+\Gamma_{0}^{2}\right]^{2}}
$$

where $\omega_{p}^{2}=4 \pi \mathrm{Ne}^{2} / \mathrm{m}, L=\left(\left(n^{2}+2\right) / 3\right)^{2}$ (Lorentz-Lorenz local field correction), e is an electron charge, $m$ an electron mass, $N$ the number of ions per $\mathrm{cm}^{3}$, $f$ a oscillator strength, and $\omega_{0}$ a center frequency of the transition.

In the equation (7), the value of oscillator strength $f$ are assumed to be the same as the case of Co doped YGaG reported by Pappalardo et al. ${ }^{\text {(21 }}$ and center frequencies of the transitions are determined from the $\epsilon_{1}^{\prime}$ spectra.

The quantity of co divalent ions in the tetrahedral site is derived from the value of total Co content measured by an EPMA and the site preference coefficient $(=20 \%)$ previously reported by Geller et al. ${ }^{[7]}, \Delta$ and $\Gamma_{0}$ are obtained through the parameter-fitting procedure of the theoretical curve to the measured data.

The result at $1.45 \mu \mathrm{m}(0.86 \mathrm{eV})$, is shown in Fig. 3. It is derived under the assumption of $f$ is equal to $2 \times 10^{-5}$ (from (2)) and $\omega_{0}$ is equal to $1.45 \mu \mathrm{m}(0.86 \mathrm{eV}$ ). The used and obtained parameters are shown in Table 1. Except for the sample 1, $\Delta$ and $\Gamma_{0}$ remain constant for increasing Co concentration. Sample 1 has smaller value of $\Delta$ than the other. It may be attributed to saturation of the Co ${ }^{2+}$ concentration in the tetrahedral site. Also, the charge compensation is incomplete in sample 1 , since Ge concentration is much smaller than Co concentration. In such a case, the effect of tetrahedral $\mathrm{Co}^{3+}$ should be taken into consideration. From these results, it is concluded that magneto-optical effect and absorption at $1.5 \mu \mathrm{m}$ band are essentially decided by the content of Co divalent ions in the tetrahedral sites. Therefore, when the co concentration is increased, the value of magneto-optical figure of merit of Co doped iron garnet films becomes almost constant against co content.

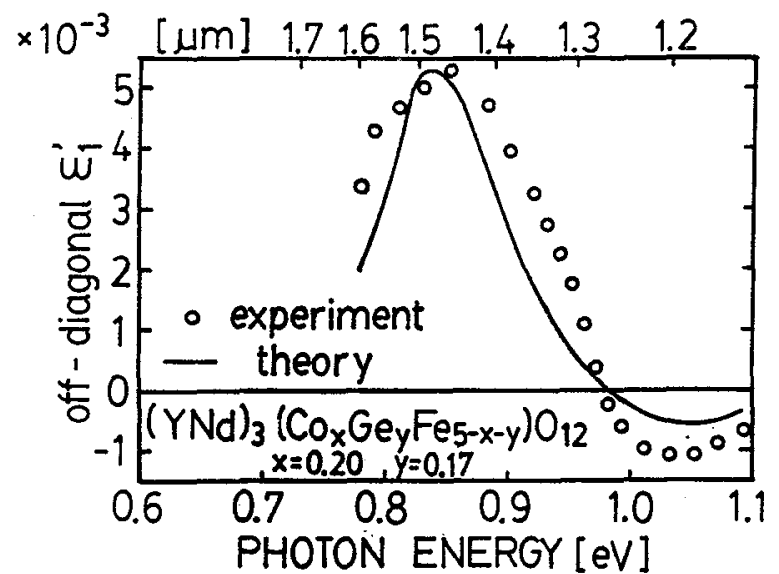

Fig. 3. $\epsilon i$ spectrum and the result of the fitting of theoretical curve (for the sample 2 in Table 1) using $\Delta$ and $\Gamma_{0}$ as fitting parameters for the Lorentz's formula in which oniy one transition is taken into consideration.
Table 1. Obtained parameters from the fitting procedure together with material parameters. Only one transition is assumed. The oscillator strength $f$ and the center frequency $\omega_{0}$ are assumed as $f=2 \times 10^{-5}$ and $\omega_{0}=1.45 \mathrm{um}(0.86 \mathrm{eV})$, respectivity. In sample $1, \Delta$ has a smaller value than the other. It may be attributed to saturation of $\mathrm{Co}^{2+}$ content in the tetrahedral sites (see text).

\begin{tabular}{cccccc}
\hline Sample & Co & Ge & $\Gamma_{0}\left[\mathrm{~cm}^{-1}\right] \Delta\left[\mathrm{cm}^{-1}\right](\varepsilon \text { i })_{\text {MAX }} \times 10^{-3}$ \\
\hline 1 & 0.81 & 0.64 & 1050 & 633 & 10.0 \\
2 & 0.20 & 0.17 & 1050 & 1486 & 5.2 \\
3 & 0.14 & 0.15 & 1050 & 1478 & 4.3 \\
4 & 0.09 & 0.08 & 1050 & 1443 & 2.1 \\
5 & 0.05 & 0.06 & 1050 & 1443 & 1.5 \\
\hline
\end{tabular}

In Fig. 3, the theoretical curve is derived under an assumption of existence of only one transition at $1.45 \mu \mathrm{m}(0.86 \mathrm{eV})$, The calculated line shape does not fit well in detail with the experimental data.

Saito et al. ${ }^{[8]}$ reported a theoretical analysis of the effects of crystal field due to trigonal distortion of the tetrahedron to $\epsilon_{1}^{\prime}$ spectrum. They concluded that $\epsilon_{i}^{\prime}$ spectrum about $1.5 \mu m$ consists of three transitions. We attempted to derive $\Delta$ and $\Gamma_{0}$ from their theory as follows. From Fig. 3, center frequencies of transitions are assumed as the frequencies at the shoulders in the curve and values of the oscillator strength are assumed to be the same as the data given by Pappalardo et al.. They are shown in Table 2. Using these data, $\Delta$ and $\Gamma_{0}$ for each transition are derived by parameter-fitting. An example of result is shown in Table 2 and Fig. 4 . In comparison with Fig. 3 , the fitted curve reproduce the experimental one much better. Again $\Delta$ and $\Gamma_{0}$ are constant against the change of Co concentration. 


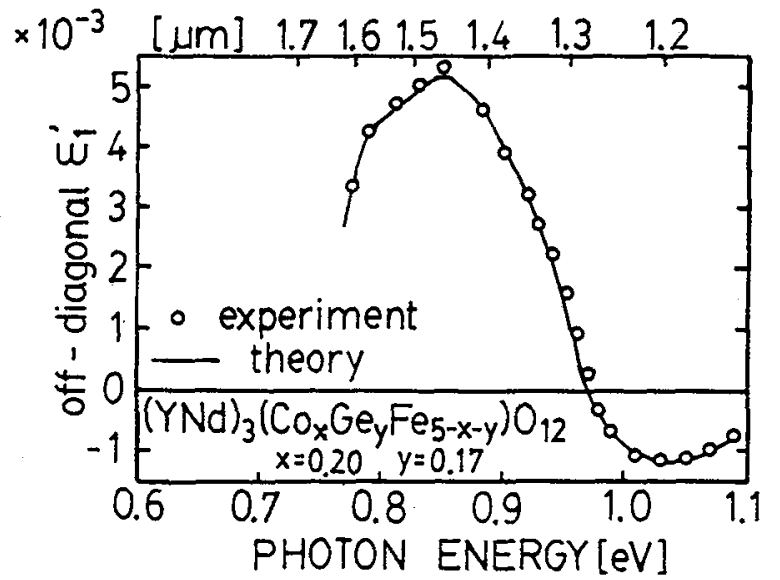

Fig. 4. An example of theoretical curve derived from the Saito's theory in which three transitions are taken into acount. The obtained curve is fitted very well with the experimental data. Used and obtained parameters are shown in the Table.2.

Saito et aT. ${ }^{[9]}$ also reported recently the influence of co trivalent ion in distorted tetrahedral site on magneto-optical effect considering $\mathrm{S}_{4}$ symmetry. It was predicted that spectrum shows a dispersive behavior at $1.3 \mu \mathrm{m}$ $(0.95 \mathrm{eV})$. Fig. 5 shows measured $\epsilon i^{\prime}$ and $\epsilon \boldsymbol{f}^{\prime \prime}$ spectra in $\mathrm{CO}^{3+}$ doped $\mathrm{YbSmIG}$ grown from a $\mathrm{PbO}-$ $\mathrm{V}_{2} \mathrm{O}_{5}$ fluxed melt. The peak frequency of $\epsilon_{i}^{\prime}$ is appeared at a little higher energy than in the divalent co case. It is not so easy to derive acculate parameters $\left(\Delta, \Gamma_{0}\right)$ through the parameter fitting procedure, because there exist an influence of divalent $C_{0}$ on $\epsilon_{1}^{\prime}$ and $\epsilon_{1}^{\prime \prime}$ around 1.3um. Further analysis by the theory by Saito et al. ${ }^{[9]}$ is now proceeding.
Table 2. Obtained parameters from the fitting procedure for the sample 2 of Table 1 by using the theory developed by Saito et a]. ${ }^{[8]}$ assuming the exsistence of three transitions caused by distorted tetrahedral site. The value of oscillator strength are assumed to be the same as the case in the reference [2].

\begin{tabular}{llccc}
\hline \multicolumn{1}{c}{$\omega_{0}$} & $\mathrm{f} \times 10^{-5}$ & $\Gamma_{0}\left[\mathrm{~cm}^{-1}\right]$ & $\Delta\left[\mathrm{cm}^{-1}\right]$ \\
\hline $\mathrm{eV}]$ & {$[\mu \mathrm{m}]$} & & & \\
\hline 0.78 & 1.58 & 0.20 & 470 & 1480 \\
0.85 & 1.46 & 2.00 & 850 & 990 \\
0.92 & 1.35 & 29.00 & 590 & 15 \\
\hline
\end{tabular}

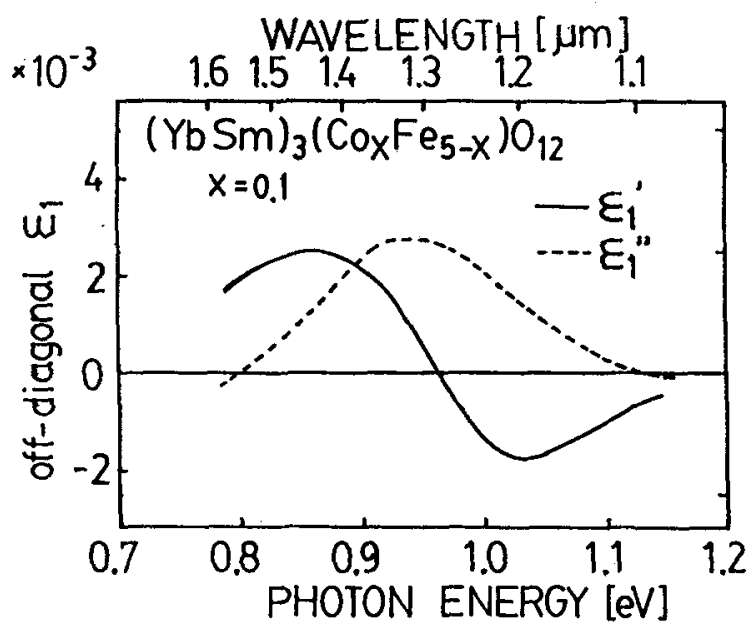

Fig. 5. Measured off-diagonal elements of dielectric tensor of trivalent Co doped YbSmIG. Film composition is $\mathrm{Yb}_{1 .}{ }_{15} \mathrm{SE}_{1.85} \mathrm{Co0}_{1 .} \mathrm{Fe}_{4.9} \mathrm{O}_{12}$

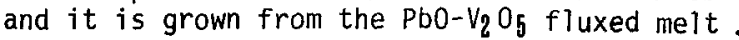

\section{CONCLUSIONS}

Dielectric tensor of Co-substituted iron garnet films grown by LPE method were shown. Obtained results are as follows,

1. Splitting width $\Delta$ and half-width at half-height of the transition $\Gamma_{0}$ are constant against Co content. Then, the value of magneto-optical effect and optical absorption at $1.5 \mu \mathrm{m}$ band are essentially determined by the amount of $\mathrm{Co}^{2+}$ ions in the tetrahedral sites. Figure of merit is therefore constant against co content.

2. The magneto-optical effect at $1.5 \mu \mathrm{m}(0.8 \mathrm{eV})$ caused by three optical transitions.

3. $\epsilon_{i}$ and $\epsilon_{1}$ " spectra of trivalent co doped films are shown.

Acknowledgments The authors wish to thank Dr. K. Sato of Tokyo University of Agriculture and Technology and Dr. T. Tsushima, Dr. K. Shinagawa, and Dr. T. Saito of Toho University for enlightening discussion on interpretation of the experimental results.

\section{References}

[1] K. Egashira and T. Manabe :IEEE Trans. Magn. MAG-8 646 (1972).

[2] R. Papparaldo, D. L. Wood, and R. C. Linares Jr.:J. Chem. Phys. 352047 (1961).

[3] D. M. Roessler :Brit. J. Appl. Phys. 161119 (1965).

[4] S. Wittecock, T. J. A. Popma, J. M. Robertson, and P. F. Bongers :Phys. Rev. 122777 (1975).

[5] F. J. Kahn, P. S. Pershan, and J. P. Remeika :Phys. Rev. 186891 (1969)

[6] K. Sato :Jpn. J. Appl. Phys. 202403 (1981).

[7] S. Geller, H. J. Williams, G. P. Espinosa, and R. C. Sherwood :Phys. Rev. 136 A1650 (1964).

[8] T. Saito, H. Hamanaka, K. Shinagawa, and T. Tsushima :MSJ conf. 7aB-1 (1986).

[9] T. Saito, K. Tamanoi, K. Shinagawa, and T. Tsushima :ISMO '87 20A-02 (1987). 